\title{
Formulas and equations for finding scattering data from the Dirichlet-to-Neumann map with nonzero background potential
}

\author{
R. G. Novikov \\ CNRS, Laboratoire de Mathématiques Jean Leray (UMR 6629), Université de Nantes, BP 92208, \\ F-44322, Nantes cedex 03, France \\ e-mail: novikov@math.univ-nantes.fr
}

\begin{abstract}
For the Schrödinger equation at fixed energy with a potential supported in a bounded domain we give formulas and equations for finding scattering data from the Dirichletto-Neumann map with nonzero background potential. For the case of zero background potential these results were obtained in [R.G.Novikov, Multidimensional inverse spectral problem for the equation $-\Delta \psi+(v(x)-E u(x)) \psi=0$, Funkt. Anal. i Ego Prilozhen 22(4), pp.11-22, (1988)].
\end{abstract}

\section{Introduction}

Consider the Schrödinger equation

$$
-\Delta \psi+v(x) \psi=E \psi, \quad x \in D
$$

where

$$
\begin{aligned}
& D \text { is an open bounded domain in } \mathbb{R}^{d}, \quad d \geq 2 \text {, } \\
& \text { with } \partial D \in C^{2}, \\
& \qquad v \in L^{\infty}(D) .
\end{aligned}
$$

We also assume that

$$
\begin{aligned}
& E \text { is not a Dirichlet eigenvalue for } \\
& \text { the operator }-\Delta+v \text { in } D \text {. }
\end{aligned}
$$

Consider the map $\Phi(E)$ such that

$$
\left.\frac{\partial \psi}{\partial \nu}\right|_{\partial D}=\Phi(E)\left(\left.\psi\right|_{\partial D}\right)
$$

for all sufficiently regular solutions of (1.1) in $\bar{D}=D \cup \partial D$, for example, for all $\psi \in H^{1}(D)$ satisfying (1.1), where $\nu$ is the outward normal to $\partial D$. The map $\Phi(E)$ is called the Dirichlet-to-Neumann map for equation (1.1).

Consider the Schrödinger equation

$$
-\Delta \psi+v(x) \psi=E \psi, \quad x \in \mathbb{R}^{d},
$$

where

$$
\rho^{d+\varepsilon} v \in L^{\infty}\left(\mathbb{R}^{d}\right), \quad d \geq 2, \text { for some } \varepsilon>0,
$$


where $\rho$ denotes the multiplication operator by the function $\rho(x)=1+|x|$. For equation (1.5) we consider the functions $\psi^{+}$and $f$ of the classical scattering theory and the Faddeev functions $\psi, h, \psi_{\gamma}, h_{\gamma}$ (see, for example, [F1], [F2], [F3], [HN], [Ne]).

The functions $\psi^{+}$and $f$ are defined as follows:

$$
\begin{aligned}
& \psi^{+}(x, k)=e^{i k x}+\int_{\mathbb{R}^{d}} G^{+}(x-y, k) v(y) \psi^{+}(y, k) d y \\
& G^{+}(x, k)=-\left(\frac{1}{2 \pi}\right)^{d} \int_{\mathbb{R}^{d}} \frac{e^{i \xi x} d \xi}{\xi^{2}-k^{2}-i 0},
\end{aligned}
$$

where $x, k \in \mathbb{R}^{d}, k^{2}>0$ (and at fixed $k$ the formula (1.7) is an equation for $\psi^{+}$in $L^{\infty}\left(\mathbb{R}^{d}\right)$ );

$$
f(k, l)=\left(\frac{1}{2 \pi}\right)^{d} \int_{\mathbb{R}^{d}} e^{-i l x} v(x) \psi^{+}(x, k) d x
$$

where $k, l \in \mathbb{R}^{d}, k^{2}>0$. Here $\psi^{+}(x, k)$ satisfies (1.5) for $E=k^{2}$ and describes scattering of the plane waves $e^{i k x} ; f(k, l), k^{2}=l^{2}$, is the scattering amplitude for equation (1.5) for $E=k^{2}$. The equation (1.7) is called the Lippman-Schwinger integral equation.

The functions $\psi$ and $h$ are defined as follows:

$$
\begin{aligned}
& \psi(x, k)=e^{i k x}+\int_{\mathbb{R}^{d}} G(x-y, k) v(y) \psi(y, k) d y \\
& G(x, k)=e^{i k x} g(x, k), \quad g(x, k)=-\left(\frac{1}{2 \pi}\right)^{d} \int_{\mathbb{R}^{d}} \frac{e^{i \xi x} d \xi}{\xi^{2}+2 k \xi}
\end{aligned}
$$

where $x \in \mathbb{R}^{d}, k \in \mathbb{C}^{d} \backslash \mathbb{R}^{d}$ (and at fixed $k$ the formula (1.10) is an equation for $\psi=$ $e^{i k x} \mu(x, k)$, where $\mu$ is sought in $\left.L^{\infty}\left(\mathbb{R}^{d}\right)\right)$;

$$
h(k, l)=\left(\frac{1}{2 \pi}\right)^{d} \int_{\mathbb{R}^{d}} e^{-i l x} v(x) \psi(x, k) d x
$$

where $k, l \in \mathbb{C}^{d} \backslash \mathbb{R}^{d}, \operatorname{Im} k=\operatorname{Im} l$. Here $\psi(x, k)$ satisfies (1.5) for $E=k^{2}$, and $\psi, G$ and $h$ are (nonanalytic) continuations of $\psi^{+}, G^{+}$and $f$ to the complex domain. In particular, $h(k, l)$ for $k^{2}=l^{2}$ can be considered as the "scattering" amplitude in the complex domain for equation (1.5) for $E=k^{2}$. The functions $\psi_{\gamma}$ and $h_{\gamma}$ are defined as follows:

$$
\psi_{\gamma}(x, k)=\psi(x, k+i 0 \gamma), \quad h_{\gamma}(k, l)=h(k+i 0 \gamma, l+i 0 \gamma),
$$

where $x, k, l, \gamma \in \mathbb{R}^{d},|\gamma|=1$. Note that

$$
\psi^{+}(x, k)=\psi_{k /|k|}(x, k), \quad f(k, l)=h_{k /|k|}(k, l),
$$

where $x, k, l \in \mathbb{R}^{d},|k|>0$.

We consider $f(k, l)$ and $h_{\gamma}(k, l)$, where $k, l, \gamma \in \mathbb{R}^{d}, k^{2}=l^{2}=E, \gamma^{2}=1$, and $h(k, l)$, where $k, l \in \mathbb{C}^{d} \backslash \mathbb{R}^{d}, \operatorname{Im} k=\operatorname{Im} l, k^{2}=l^{2}=E$, as scattering data $S_{E}$ for equation (1.5) at 
fixed $E \in] 0,+\infty\left[\right.$. We consider $h(k, l)$, where $k, l \in \mathbb{C}^{d} \backslash \mathbb{R}^{d}, \operatorname{Im} k=\operatorname{Im} l, k^{2}=l^{2}=E$, as scattering data $S_{E}$ for equation (1.5) at fixed $\left.E \in \mathbb{C} \backslash\right] 0,+\infty[$.

Let $D$ be a fixed domain satisfying (1.2a). Let

$$
v \in L^{\infty}(D) \text { and } v \equiv 0 \text { on } \mathbb{R}^{d} \backslash \bar{D} \text {. }
$$

For $v$ of (1.15) we consider the Dirichlet-to-Neumann map $\Phi(E)$ for equation (1.1) and the scattering data $S_{E}$ for equation (1.5).

In the present work we continue studies of [No1] on the following inverse boundary value problem for equation (1.1):

Problem 1. Find $v$ (in (1.1)) from $\Phi(E)$ (where $E$ is fixed or belongs to some set).

More precisely, we develop formulas and equations of [No1] which reduce Problem 1 to the following inverse scattering problem for equation (1.5):

Problem 2. Find $v$ (in (1.5)) from $S(E)$ (where $E$ is fixed or belongs to some set).

Concerning results given in the literature on Problem 1, see [SU], [No1], [A], [NSU], [Na1], [Na2], $[\mathrm{M}]$ and references therein. Concerning results given in the literature on Problem 2, see $[\mathrm{BC}],[\mathrm{HN}],[\mathrm{No} 2],[\mathrm{No} 3],[\mathrm{IS}],[\mathrm{GN}],[\mathrm{No} 4],[\mathrm{No} 5],[\mathrm{E}],[\mathrm{Ch}],[\mathrm{BBMRS}]$, $[\mathrm{BMR}]$ and references therein.

The main results of the present work consist of Theorem 1 and Propositions 1 and 2 of Section 2. In these results we consider for fixed $E$ two potentials $v_{0}$ and $v$ satisfying (1.15) and (1.3). In Theorem 1 and Proposition 1 we give formulas and equations for finding $S_{E}$ from $\Phi(E)-\Phi_{0}(E)$ and from (some functions found from) $v_{0}$, where $S_{E}$ and $\Phi(E)$ correspond to $v$ and $\Phi_{0}(E)$ corresponds to $v_{0}$. In Proposition 2 we give a result on the solvability of equations of Theorem 1 .

For the case when $v_{0} \equiv 0$, Theorem 1 and Propositions 1 and 2 were obtained for the first time in [No1] (see also [HN], [Na1], [Na2]), where using these results Problem 1 was reduced to Problem 2. For the case when the best known approximation $v_{0}$ to $v$ of Problem 1 is not identically zero (and especially when $v_{0}$ is really close to $v$ ), results of the present work reduce Problem 1 to Problem 2 in a more stable way than it was done in $[\mathrm{No1}$.

Note that generalizing results of [No1] to the case of nonzero background potential $v_{0}$ we used essentially results of [No4]. The main results of the present work are presented in detail in Section 2.

Note, finally, that by the present work we start a development of new exact reconstruction algorithms for Problem 1.

\section{Main results}

To formuate our results we need to introduce some additional notations. Consider (under the assumption (1.6)) the sets $\mathcal{E}, \mathcal{E}_{\gamma}, \mathcal{E}^{+}$defined as follows:

$$
\begin{gathered}
\mathcal{E}=\left\{\zeta \in \mathbb{C}^{d} \backslash \mathbb{R}^{d}: \text { equation }(1.10) \text { for } k=\zeta\right. \text { is not } \\
\text { uniquely solvable for } \left.\psi=e^{i k x} \mu \text { with } \mu \in L^{\infty}\left(\mathbb{R}^{d}\right)\right\}, \\
\mathcal{E}_{\gamma}=\left\{\zeta \in \mathbb{R}^{d} \backslash 0: \text { equation }(1.10) \text { for } k=\zeta+i 0 \gamma\right. \text { is not } \\
\text { uniquely solvable for } \left.\psi \in L^{\infty}\left(\mathbb{R}^{d}\right)\right\}, \quad \gamma \in \mathbb{S}^{d-1},
\end{gathered}
$$




$$
\begin{aligned}
& \mathcal{E}^{+}=\left\{\zeta \in \mathbb{R}^{d} \backslash 0 \text { : equation }(1.7) \text { for } k=\zeta\right. \text { is not } \\
& \text { uniquely solvable for } \left.\psi^{+} \in L^{\infty}\left(\mathbb{R}^{d}\right)\right\} .
\end{aligned}
$$

Note that $\mathcal{E}^{+}$is a well-known set of the classical scattering theory for equation (1.5) and that $\mathcal{E}^{+}=\emptyset$ for real-valued $v$ satisfying (1.6) (see, for example, [Ne]). The sets $\mathcal{E}$ and $\mathcal{E}_{\gamma}$ were considered for the first time in [F1], [F2], [F3]. Concerning the properties of $\mathcal{E}$ and $\mathcal{E}_{\gamma}$, see [F3], [HN], [LN], [Ne], [We], [Na2], [No4], [No6].

Consider (under the assumptions (1.6)) the functions $R, R_{\gamma}, R^{+}$defined as follows:

$$
R(x, y, k)=G(x-y, k)+\int_{\mathbb{R}^{d}} G(x-z, k) v(z) R(z, y, k) d z,
$$

where $x, y \in \mathbb{R}^{d}, k \in \mathbb{C}^{d} \backslash \mathbb{R}^{d}, G$ is defined by (1.11), and at fixed $y$ and $k$ the formula (2.2) is an equation for

$$
R(x, y, k)=e^{i k(x-y)} r(x, y, k),
$$

where $r$ is sought with the properties

$$
\begin{gathered}
r(\cdot, y, k) \text { is continuous on } \mathbb{R}^{d} \backslash y, \\
r(x, y, k) \rightarrow 0 \text { as }|x| \rightarrow \infty, \\
r(x, y, k)=O\left(|x-y|^{2-d}\right) \text { as } x \rightarrow y \text { for } d \geq 3, \\
r(x, y, k)=O(|\ln | x-y||) \text { as } x \rightarrow y \text { for } d=2 ; \\
R_{\gamma}(x, y, k)=R(x, y, k+i 0 \gamma),
\end{gathered}
$$

where $x, y \in \mathbb{R}^{d}, k \in \mathbb{R}^{d} \backslash 0, \gamma \in \mathbb{S}^{d-1}$;

$$
R^{+}(x, y, k)=R_{k /|k|}(x, y, k),
$$

where $x, y \in \mathbb{R}^{d}, k \in \mathbb{R}^{d} \backslash 0$. Note that $R(x, y, k), R_{\gamma}(x, y, k)$ and $R^{+}(x, y, k)$ (for their domains of definition in $k$ and $\gamma$ ) satisfy the equation

$$
(\Delta+E-v(x)) R(x, y, k)=\delta(x-y), \quad x \in \mathbb{R}^{d}, \quad y \in \mathbb{R}^{d}, \quad E=k^{2} .
$$

The function $R^{+}(x, y, k)$ (defined by means of (2.2) for $k \in \mathbb{R}^{d} \backslash 0$ with $G$ replaced by $G^{+}$of (1.8)) is well-known in the scattering theory for equations (1.5), (2.7). In particular, this function describes scattering of the spherical waves $G^{+}(x-y, k)$ generated by a source at $y$. Apparently, the functions $R$ and $R_{\gamma}$ were considered for the first time in [No5].

Note that under the assumption (1.6): equation (2.2) at fixed $y$ and $k$ is uniquely solvable for $R$ with the properties (2.3), (2.4) if and only if $k \in \mathbb{C}^{d} \backslash\left(\mathbb{R}^{d} \cup \mathcal{E}\right)$; equation (2.2) with $k=\zeta+i 0 \gamma, \zeta \in \mathbb{R}^{d} \backslash 0, \gamma \in \mathbb{S}^{d-1}$, at fixed $y, \zeta$ and $\gamma$ is uniquely solvable for $R_{\gamma}$ if and only if $\zeta \in \mathbb{R}^{d} \backslash\left(0 \cup \mathcal{E}_{\gamma}\right)$; equation (2.2) with $k=\zeta+i 0 \zeta /|\zeta|, \zeta \in \mathbb{R}^{d} \backslash 0$, at fixed $y$ and $\zeta$ is uniquely solvable for $R^{+}$if and only if $\zeta \in \mathbb{R}^{d} \backslash\left(0 \cup \mathcal{E}^{+}\right)$.

For $v$ of (1.15) we consider the map $\Phi(E)$ defined by means of (1.4), the functions $\psi^{+}, f, \psi, h, \psi_{\gamma}, h_{\gamma}$ and $R^{+}, R, R_{\gamma}$ defined by means of (1.7)-(1.13) and (2.2)-(2.6) and 
the sets $\mathcal{E}, \mathcal{E}_{\gamma}, \mathcal{E}^{+}$defined by (2.1). The Schwartz kernel of the integral operator $\Phi(E)$ will be denoted by $\Phi(x, y, E)$, where $x, y \in \partial D$.

Theorem 1. Let $D$ satisfying (1.2a) and $E$ be fixed. Let $v^{0}$ and $v$ be two potentials satisfying (1.15), (1.3). Let $\Phi, \psi^{+}, f, \psi, h, \psi_{\gamma}, h_{\gamma}, R^{+}, R, R_{\gamma}, \mathcal{E}, \mathcal{E}_{\gamma}, \mathcal{E}^{+}$correspond to $v$ (as defined above) and $\Phi^{0}, \psi^{+, 0}, f^{0}, \psi^{0}, h^{0}, \psi_{\gamma}^{0}, h_{\gamma}^{0}, R^{+, 0}, R^{0}, R_{\gamma}^{0}, \mathcal{E}^{0}, \mathcal{E}_{\gamma}^{0}, \mathcal{E}^{+, 0}$ correspond to $v^{0}$ (as defined above with $v=v^{0}$ ). Then the following formulas hold:

$$
h(k, l)=h^{0}(k, l)+\left(\frac{1}{2 \pi}\right)^{d} \int_{\partial D} \int_{\partial D} \psi^{0}(x,-l)\left(\Phi-\Phi^{0}\right)(x, y, E) \psi(y, k) d y d x
$$

for $k, l \in \mathbb{C}^{d} \backslash\left(\mathbb{R}^{d} \cup \mathcal{E}^{0} \cup \mathcal{E}\right), \operatorname{Im} k=\operatorname{Im} l, k^{2}=l^{2}=E$,

$$
\begin{aligned}
& \psi(x, k)=\psi^{0}(x, k)+\int_{\partial D} A(x, y, k) \psi(y, k) d y, \quad x \in \partial D \\
& A(x, y, k)=\int_{\partial D} R^{0}(x, z, k)\left(\Phi-\Phi^{0}\right)(z, y, E) d z, \quad x, y \in \partial D
\end{aligned}
$$

for $k \in \mathbb{C}^{d} \backslash\left(\mathbb{R}^{d} \cup \mathcal{E}^{0} \cup \mathcal{E}\right), k^{2}=E ;$

$$
h_{\gamma}(k, l)=h_{\gamma}^{0}(k, l)+\left(\frac{1}{2 \pi}\right)^{d} \int_{\partial D} \int_{\partial D} \psi_{-\gamma}^{0}(x,-l)\left(\Phi-\Phi^{0}\right)(x, y, E) \psi_{\gamma}(y, k) d y d x
$$

for $k, l \in \mathbb{R}^{d} \backslash\left(0 \cup \mathcal{E}_{\gamma}^{0} \cup \mathcal{E}_{\gamma}\right), \gamma \in \mathbb{S}^{d-1}, k^{2}=l^{2}=E, k \gamma=l \gamma$,

$$
\begin{aligned}
& \psi_{\gamma}(x, k)=\psi_{\gamma}^{0}(x, k)+\int_{\partial D} A_{\gamma}(x, y, k) \psi_{\gamma}(y, k) d y, \quad x \in \partial D \\
& A_{\gamma}(x, y, k)=\int_{\partial D} R_{\gamma}^{0}(x, z, k)\left(\Phi-\Phi^{0}\right)(z, y, E) d z, \quad x, y \in \partial D
\end{aligned}
$$

for $k \in \mathbb{R}^{d} \backslash\left(0 \cup \mathcal{E}_{\gamma}^{0} \cup \mathcal{E}_{\gamma}\right), \gamma \in \mathbb{S}^{d-1}, k^{2}=E ;$

$$
f(k, l)=f^{0}(k, l)+\left(\frac{1}{2 \pi}\right)^{d} \int_{\partial D} \int_{\partial D} \psi^{+, 0}(x,-l)\left(\Phi-\Phi^{0}\right)(x, y, E) \psi^{+}(y, k) d y d x
$$

for $k, l \in \mathbb{R}^{d} \backslash\left(0 \cup \mathcal{E}^{+, 0} \cup \mathcal{E}^{+}\right), k^{2}=l^{2}=E$,

$$
\begin{aligned}
& \psi^{+}(x, k)=\psi^{+, 0}(x, k)+\int_{\partial D} A^{+}(x, y, k) \psi^{+}(y, k) d y, \quad x \in \partial D \\
& A^{+}(x, y, k)=\int_{\partial D} R^{+}(x, z, k)\left(\Phi-\Phi^{0}\right)(z, y, E) d z, \quad x, y \in \partial D
\end{aligned}
$$

for $k \in \mathbb{R}^{d} \backslash\left(0 \cup \mathcal{E}^{+, 0} \cup \mathcal{E}^{+}\right), k^{2}=E$.

Note that in Theorem $1 d x$ and $d y$ denote the standard measure on $\partial D$ in $\mathbb{R}^{d}$. 
Note that in the formula $(2.10)$ for $h_{\gamma}(k, l)$ there is the additional restriction: $k \gamma=l \gamma$. To extend (2.10) to the general case, consider $\psi_{\gamma}(x, k, l)$ defined as follows:

$$
\begin{aligned}
& \psi_{\gamma}(x, k, l)=e^{i l x}+\int_{\mathbb{R}^{d}} G_{\gamma}(x-y, k) v(y) \psi_{\gamma}(y, k, l) d y \\
& G_{\gamma}(x, k)=G(x, k+i 0 \gamma)
\end{aligned}
$$

where $x, k, l \in \mathbb{R}^{d}, k^{2}=l^{2}>0, \gamma \in \mathbb{S}^{d-1}$ and (2.14) at fixed $\gamma, k, l$ is an equation for $\psi_{\gamma}(\cdot, k, l)$ in $L^{\infty}\left(\mathbb{R}^{d}\right)$.

Proposition 1. Let the assumptions of Theorem 1 be valid. In addition, let $\psi_{\gamma}(x, k, l)$ correspond to $v$ and $\psi_{\gamma}^{0}(x, k, l)$ correspond to $v^{0}$. Then

$$
h_{\gamma}(k, l)=h_{\gamma}^{0}(k, l)+\left(\frac{1}{2 \pi}\right)^{d} \int_{\partial D} \int_{\partial D} \psi_{-\gamma}^{0}(x,-k,-l)\left(\Phi-\Phi^{0}\right)(x, y, E) \psi_{\gamma}(y, k) d y d x
$$

for $\gamma \in \mathbb{S}^{d-1}, k \in \mathbb{R}^{d} \backslash\left(0 \cup \mathcal{E}_{\gamma}^{0} \cup \mathcal{E}_{\gamma}\right), l \in \mathbb{R}^{d}, k^{2}=l^{2}=E$.

Note that (see [F3], [No4])

$$
G_{\gamma}(x, k)=G_{\gamma}(x, l) \text { for } x, k, l \in \mathbb{R}^{d}, \quad \gamma \in \mathbb{S}^{d-1}, \quad k^{2}=l^{2}>0, \quad k \gamma=l \gamma .
$$

Therefore,

$$
\psi_{\gamma}(x, k, l)=\psi_{\gamma}(x, l, l)=\psi_{\gamma}(x, l) \text { for } x, k, l \in \mathbb{R}^{d}, \quad \gamma \in \mathbb{S}^{d-1}, \quad k^{2}=l^{2}>0, \quad k \gamma=l \gamma
$$

Therefore, (2.15), under the additional restriction $k \gamma=l \gamma$ is reduced to (2.10).

Suppose that $v$ is unknown, but $v^{0}$ and $\Phi(E)-\Phi^{0}(E)$ are known $\left(v^{0}\right.$ is considered as the best known approximation to $v$ ). Then Theorem 1 and Proposition 1 (and equations and formulas (1.7)-(1.13), (2.2)-(2.6), (2.14) for finding $\psi^{+, 0}, f^{0}, \psi^{0}, h^{0}, \psi_{\gamma}^{0}, h_{\gamma}^{0}, R^{+, 0}, R^{0}$, $R_{\gamma}^{0}$ from $v^{0}$ ) give a method for finding the scattering data $S_{E}$ (defined in the introduction) for $v$ from the background potential $v^{0}$ and the difference $\Phi(E)-\Phi^{0}(E)$. In addition, (2.9a), (2.11a), (2.13a) at fixed $k$ and $\gamma$ are linear integral equations for finding $\psi, \psi_{\gamma}, \psi^{+}$ on $\partial D$ from $\psi^{0}, \psi_{\gamma}^{0}, \psi^{+, 0}$ on $\partial D$ and $A, A_{\gamma}, A^{+}$on $\partial D \times \partial D$ (where $A, A_{\gamma}, A^{+}$are given by $(2.9 \mathrm{~b}),(2.11 \mathrm{~b}),(2.13 \mathrm{~b}))$.

Proposition 2. Under the assumptions of Theorem 1, equation (2.9a) at fixed $k \in$ $\mathbb{C}^{d} \backslash\left(\mathbb{R}^{d} \cup \mathcal{E}^{0}\right), k^{2}=E$, equation (2.11a) at fixed $\gamma \in \mathbb{S}^{d-1}$ and $k \in \mathbb{R}^{d} \backslash\left(0 \cup \mathcal{E}_{\gamma}^{0}\right), k^{2}=E$, and equation $(2.13 \mathrm{a})$ at fixed $k \in \mathbb{R}^{d} \backslash\left(0 \cup \mathcal{E}^{+, 0}\right)$ are Fredholm linear integral equations of the second kind for $\psi, \psi_{\gamma}$ and $\psi^{+}$(respectively) in $L^{\infty}(\partial D)$ and are uniquely solvable (in this space) if and only if $k \notin \mathcal{E}$ for (2.9a), $k \notin \mathcal{E}_{\gamma}$ for (2.11a) and $k \notin \mathcal{E}^{+}$for (2.13a).

Note that

$$
\psi(x, k)=e^{i k x} \mu(x, k), \quad \psi^{0}(x, k)=e^{i k x} \mu^{0}(x, k), \quad x \in \mathbb{R}^{d}, \quad k \in \mathbb{C}^{d} \backslash \mathbb{R}^{d},
$$

where $e^{i k x}$ is an exponentially increasing factor and

$$
\mu(x, k) \rightarrow 1, \quad \mu^{0}(x, k) \rightarrow 1 \text { as }|k| \rightarrow \infty
$$


for $k^{2}=E$ at fixed $E$, where $|k|=\sqrt{(R e k)^{2}+(\operatorname{Im} k)^{2}}$. Therefore, it is convenient to write equation (2.9) of Theorem 1 as follows:

$$
\begin{aligned}
& \mu(x, k)=\mu^{0}(x, k)+\int_{\partial D} B(x, y, k) \mu(y, k) d y, \quad x \in \partial D \\
& B(x, y, k)=\int_{\partial D} r^{0}(x, z, k) e^{-i k z}\left(\Phi-\Phi^{0}\right)(z, y, E) d z e^{i k y}, \quad x, y \in \partial D
\end{aligned}
$$

for $k \in \mathbb{C}^{d} \backslash\left(\mathbb{R}^{d} \cup \mathcal{E}^{0} \cup \mathcal{E}\right)$, where $r^{0}$ and $R^{0}$ are related by $(2.3)$.

Theorem 1 and Proposition 1 reduce Problem 1 to Problem 2 (these problems are formulated in the introduction).

For the case when $v^{0} \equiv 0$, Theorem 1 and Propositions 1 and 2 were obtained in [No1] (see also [Na1], [Na2]). Note that the basic results of [No1], in particular formula (2.8) and equation (2.9) for $v^{0} \equiv 0$ and $d=3$, were presented already in the survey given in [HN].

For the case when the best known approximation $v^{0}$ to $v$ is not identically zero (and especially when $v^{0}$ is really close to $v$ ) Theorem 1 and Proposition 1 give a more convenient (in particular, for the stability analysis) method for reducing Problem 1 to Problem 2 than in [No1]. To explain this more precisely, consider, in particular, the integral operators $B(k)$, $A_{\gamma}(k), A^{+}(k)$ (with the Schwartz kernels $B(x, y, k), A_{\gamma}(x, y, k), A^{+}(x, y, k)$ ) of equations (2.20a), (2.11a), (2.13a). To have a simple and stable (with respect to small errors in $\Phi(E)$ ) method for solving equations (2.20a) (for fixed $\left.k \in \mathbb{C}^{d} \backslash\left(\mathbb{R}^{d} \cup \mathcal{E}^{0}\right), k^{2}=E\right),(2.11 \mathrm{a}$ ) (for fixed $\gamma \in \mathbb{S}^{d-1}$ and $k \in \mathbb{R}^{d} \backslash\left(0 \cup \mathcal{E}_{\gamma}^{0}\right), k^{2}=E$ ) and (2.13a) (for fixed $k \in \mathbb{R}^{d} \backslash\left(0 \cup \mathcal{E}^{+, 0}\right)$, $\left.k^{2}=E\right)$ it is important to have that

$$
\|B(k)\|<\eta, \quad\left\|A_{\gamma}(k)\right\|<\eta, \quad\left\|A^{+}(k)\right\|<\eta,
$$

respectively, for some $\eta<1$, where $\|A\|$ is the norm of an operator $A$ (for example) in $L^{\infty}(\partial D)$. In this case equations $(2.20 \mathrm{a}),(2.11 \mathrm{a}),(2.13 \mathrm{a})$ are uniquely solvable by the method of successive approximations. In addition, if $\eta \ll 1$, then (2.20a), (2.11a), (2.13a) can be solved in the first approximation as

$$
\mu(x, k) \approx \mu^{0}(x, k), \quad \psi_{\gamma}(x, k) \approx \psi_{\gamma}^{0}(x, k), \quad \psi^{+}(x, k) \approx \psi^{+, 0}(x, k)
$$

and $h, h_{\gamma}, f$ can be determined in the first (nontrivial) approximation as

$$
h(k, l) \approx h^{0}(k, l)+\left(\frac{1}{2 \pi}\right)^{d} \int_{\partial D} \int_{\partial D} \psi^{0}(x,-l)\left(\Phi-\Phi^{0}\right)(x, y, E) \psi^{0}(y, k) d y d x
$$

for $l \in \mathbb{C}^{d} \backslash \mathbb{R}^{d}, \operatorname{Im} l=\operatorname{Im} k, l^{2}=k^{2}=E($ and for $k$ of $B(k)$ of $(2.21)$ ),

$$
h_{\gamma}(k, l) \approx h_{\gamma}^{0}(k, l)+\left(\frac{1}{2 \pi}\right)^{d} \int_{\partial D} \int_{\partial D} \psi_{-\gamma}^{0}(x,-k,-l)\left(\Phi-\Phi^{0}\right)(x, y, E) \psi_{\gamma}^{0}(y, k) d y d x
$$

for $l \in \mathbb{R}^{d} \backslash \mathcal{E}_{\gamma}^{0}, l^{2}=k^{2}=E$ (and for $k, \gamma$ of $A_{\gamma}(k)$ of $(2.21)$ ),

$$
f(k, l) \approx f^{0}(k, l)+\left(\frac{1}{2 \pi}\right)^{d} \int_{\partial D} \int_{\partial D} \psi^{+, 0}(x,-l)\left(\Phi-\Phi^{0}\right)(x, y, E) \psi^{+, 0}(y, k) d y d x
$$


for $l \in \mathbb{R}^{d}, l^{2}=k^{2}=E$ (and for $k$ of $A^{+}(k)$ of $(2.21)$ ).

Note that the direct problem of finding $\psi^{+, 0}, f^{0}, \psi^{0}, h^{0}, \psi_{\gamma}^{0}, h_{\gamma}^{0}, R^{+, 0}, R^{0}, R_{\gamma}^{0}$ (involved into (2.8)-(2.13), (2.15), (2.20)) from $v^{0}$ is (relatively) well understood (in comparison with the problem of solving $(2.20 \mathrm{a}),(2.11 \mathrm{a}),(2.13 \mathrm{a})$ without the assumptions $(2.21))$ and is sufficiently stable.

Note that in (2.20a), apparently, unfortunately, almost always

$$
\|B(k)\| \rightarrow \infty \quad \text { (exponentially fast) as }|k| \rightarrow \infty
$$

for $k \in \mathbb{C}^{d}, k^{2}=E$ at fixed $E$, in spite of (2.19).

To have $(2.21)$ for $B(k)$ for maximally large domain in $k \in \mathbb{C}^{d} \backslash \mathbb{R}^{d}, k^{2}=E$, and when $E>0$ for $A_{\gamma}(k)$ for maximally large domain in $\gamma \in \mathbb{S}^{d-1}$ and $k \in \mathbb{R}^{d}, k^{2}=E$, and for $A^{+}(k)$ for $k \in \mathbb{R}^{d}, k^{2}=E$, it is important to have that $\left\|\Phi(E)-\Phi^{0}(E)\right\|$ is as small as possible. The smallness of $\left\|\Phi(E)-\Phi^{0}(E)\right\|$ follows from the closeness of $v^{0}$ to $v$ (for example) in $L^{\infty}(D)$ for fixed $D, v$ and $E$, under the conditions (1.2), (1.3).

As soon as Problem 1 is reduced to Problem 2, one can use for solving Problem1 methods of [HN], [No2], [Na2], [IS], [No4], [No5], [E], [BBMRS], [BMR] (and further references given therein).

\section{Proofs of Theorem 1 and Propositions 1 and 2}

For the case when $v^{0} \equiv 0$, Theorem 1 and Propositions 1 and 2 were proved in [No1]. In this section we generalize these proofs of [No1] to the case of nonzero background potential $v^{0}$. To this end we use, in particular, some results of [A] and [No4].

Proof of Theorem 1. We proceed from the following formulas and equations (being valid under the assumption (1.6) on $v^{0}$ and $v$ ):

$$
h(k, l)=h^{0}(k, l)+\left(\frac{1}{2 \pi}\right)^{d} \int_{\mathbb{R}^{d}} \psi^{0}(x,-l)\left(v(x)-v^{0}(x)\right) \psi(x, k) d x
$$

for $k, l \in \mathbb{C}^{d} \backslash\left(\mathbb{R}^{d} \cup \mathcal{E}^{0} \cup \mathcal{E}\right), \operatorname{Im} k=\operatorname{Im} l, k^{2}=l^{2}$,

$$
\psi(x, k)=\psi^{0}(x, k)+\int_{\mathbb{R}^{d}} R^{0}(x, y, k)\left(v(y)-v^{0}(y)\right) \psi(y, k) d y,
$$

where $x \in \mathbb{R}^{d}, k \in \mathbb{C}^{d} \backslash\left(\mathbb{R}^{d} \cup \mathcal{E}^{0}\right)$ (and (3.2) at fixed $k$ is an equation for $\psi=e^{i k x} \mu(x, k)$, where $\mu$ is sought in $\left.L^{\infty}\left(\mathbb{R}^{d}\right)\right)$,

$$
h_{\gamma}(k, l)=h_{\gamma}^{0}(k, l)+\left(\frac{1}{2 \pi}\right)^{d} \int_{\mathbb{R}^{d}} \psi_{-\gamma}^{0}(x,-l)\left(v(x)-v^{0}(x)\right) \psi_{\gamma}(x, k) d x
$$

for $\gamma \in \mathbb{S}^{d-1}, k, l \in \mathbb{R}^{d} \backslash\left(0 \cup \mathcal{E}_{\gamma}^{0} \cup \mathcal{E}_{\gamma}\right), k^{2}=l^{2}, k \gamma=l \gamma$.

$$
\psi_{\gamma}(x, k)=\psi_{\gamma}^{0}(x, k)+\int_{\mathbb{R}^{d}} R_{\gamma}^{0}(x, y, k)\left(v(y)-v^{0}(y)\right) \psi_{\gamma}(y, k) d y
$$


where $x \in \mathbb{R}^{d}, \gamma \in \mathbb{S}^{d-1}, k \in \mathbb{R}^{d} \backslash\left(0 \cup \mathcal{E}_{\gamma}^{0}\right)$ (and (3.4) at fixed $\gamma$ and $k$ is an equation for $\psi_{\gamma}$ in $\left.L^{\infty}\left(\mathbb{R}^{d}\right)\right)$,

$$
f(k, l)=f^{0}(k, l)+\left(\frac{1}{2 \pi}\right)^{d} \int_{\mathbb{R}^{d}} \psi^{+, 0}(x,-l)\left(v(x)-v^{0}(x)\right) \psi^{+}(x, k) d x
$$

for $k, l \in \mathbb{R}^{d} \backslash\left(0 \cup \mathcal{E}^{+, 0} \cup \mathcal{E}^{+}\right), k^{2}=l^{2}$,

$$
\psi^{+}(x, k)=\psi^{+, 0}(x, k)+\int_{\mathbb{R}^{d}} R^{+, 0}(x, y, k)\left(v(y)-v^{0}(y)\right) \psi^{+}(y, k) d y
$$

where $x \in \mathbb{R}^{d}, k \in \mathbb{R}^{d} \backslash\left(0 \cup \mathcal{E}^{+, 0}\right)$ (and (3.6) at fixed $k$ is an equation for $\psi^{+}$in $L^{\infty}\left(\mathbb{R}^{d}\right)$ ). (We remind that $\psi^{+}, f, \psi, h, \psi_{\gamma}, h_{\gamma}$ were defined in the introduction by means of (1.7)(1.13).) Equation (3.6) is well-known in the classical scattering theory for the Schrödinger equation (1.5). Formula (3.5) was given, in particular, in [St]. To our knowledge formulas and equations (3.1)-(3.4) were given for the first time in [No4].

Note that, under the assumption (1.6):

(3.2) at fixed $k \in \mathbb{C}^{d} \backslash\left(\mathbb{R}^{d} \cup \mathcal{E}^{0}\right)$ is uniquely solvable for $\psi=e^{i k x} \mu(x, k)$ with $\mu \in L^{\infty}\left(\mathbb{R}^{d}\right)$ if and only if $k \notin \mathcal{E}$;

(3.4) at fixed $\gamma \in \mathbb{S}^{d-1}, \quad k \in \mathbb{R}^{d} \backslash\left(0 \cup \mathcal{E}_{\gamma}^{0}\right)$ is uniquely solvable for $\psi_{\gamma} \in L^{\infty}\left(\mathbb{R}^{d}\right)$ if and only if $k \notin \mathcal{E}_{\gamma}$;

(3.6) at fixed $k \in \mathbb{R}^{d} \backslash\left(0 \cup \mathcal{E}^{+, 0}\right)$ is uniquely solvable for $\psi^{+} \in L^{\infty}\left(\mathbb{R}^{d}\right)$ if and only if $k \notin \mathcal{E}^{+}$.

In a similar way with [No1], (under the assumptions of Theorem 1) formulas and equations (3.1)-(3.6) can be transformed into (2.8)-(2.13) by means of the following Green's formula

$$
\begin{aligned}
& \int_{D}\left(u_{1}(x) \Delta u_{2}(x)-u_{2}(x) \Delta u_{1}(x)\right) d x= \\
& \int_{\partial D}\left(u_{1}(x) \frac{\partial u_{2}(x)}{\partial \nu}-u_{2}(x) \frac{\partial u_{1}(x)}{\partial \nu}\right) d x
\end{aligned}
$$

(where $d x$ in the right-hand side of (3.8) denotes the standard measure on $\partial D$ in $\mathbb{R}^{d}$ ). (To start these transformations, we use that $\psi(x, k), \psi_{\gamma}(x, k)$ and $\psi^{+}(x, k)$ satisfy (1.5) for $E=k^{2}$ and replace $v \psi$ in $(3.1),(3.2)$ by $(\Delta+E) \psi, v \psi_{\gamma}$ in $(3.3),(3.4)$ by $(\Delta+E) \psi_{\gamma}$ and $v \psi^{+}$in $(3.5),(3.6)$ by $(\Delta+E) \psi^{+}$.) However, these calculations can be shortened by means of the following Alessandrini identity (being valid under the assumptions of Theorem 1):

$$
\int_{D}\left(v(x)-v^{0}(x)\right) \psi(x) \psi^{0}(x) d x=\int_{\partial D} \int_{\partial D} \psi^{0}(x)\left(\Phi-\Phi^{0}\right)(x, y, E) \psi(y) d y d x
$$

for any $\psi$ and $\psi^{0}$ such that $\psi$ satisfies $(1.1), \psi^{0}$ satisfies (1.1) with $v$ replaced by $v^{0}$ and where $\psi$ and $\psi^{0}$ are sufficiently regular in $D$, for example, $\psi, \psi^{0} \in H^{1}(D)$. In a slightly 
different form the identity (3.9) was given in Lemma 1 of $[\mathrm{A}]$. The proof of (3.9) is based on $(3.8)$.

Formula (2.8) follows from (3.1), (3.9) and the fact that $\psi(x, k)$ satisfies (1.5) for $E=k^{2}$ and $\psi^{0}(x,-l)$ satisfies (1.5) with $v$ replaced by $v^{0}$, for $E=l^{2}$. Formulas (2.9) with $x \in \mathbb{R}^{d} \backslash \bar{D}$ follow from (3.2) with $x \in \mathbb{R}^{d} \backslash \bar{D}$, (3.9) and the fact that $\psi(y, k)$ satisfies (1.5) in $y$ for $E=k^{2}$ and that $R^{0}(x, y, k)$ for $x \in \mathbb{R}^{d} \backslash \bar{D}$ satisfies (1.5) in $y$ in an open neighborhood of $\bar{D}$, with $v$ replaced by $v^{0}$, for $E=k^{2}$. The latter statement about $R^{0}$ follows from (2.7) and the symmetry

$$
R(x, y, k)=R(y, x,-k)
$$

where $x, y \in \mathbb{R}^{d}, k \in \mathbb{C}^{d} \backslash \mathbb{R}^{d}$ (and $R$ is defined by means of (2.2)-(2.4)). The symmetry (3.10) was found in [No4]. Finally, formulas (2.9) for $x \in \partial D$ arise as a limit of (2.9) with $x \in \mathbb{R}^{d} \backslash \bar{D}$.

The proof of (2.10)-(2.13) is similar to the proof of $(2.8),(2.9)$.

Theorem 1 is proved.

Proof of Proposition 1. In this proof we obtain and use the following formula (being valid under the assumption (1.6) on $v^{0}$ and $v$ ):

$$
h_{\gamma}(k, l)=h_{\gamma}^{0}(k, l)+\left(\frac{1}{2 \pi}\right)^{d} \int_{\mathbb{R}^{d}} \psi_{-\gamma}^{0}(x,-k,-l)\left(v(x)-v^{0}(x)\right) \psi_{\gamma}(x, k) d x
$$

for $\gamma \in \mathbb{S}^{d-1}, k \in \mathbb{R}^{d} \backslash\left(0 \cup \mathcal{E}_{\gamma}^{0} \cup \mathcal{E}_{\gamma}\right), l \in \mathbb{R}^{d}, k^{2}=l^{2}$. Formula (2.15) follows from (3.11), (3.9) and the fact that $\psi_{\gamma}(x, k)$ satisfies $(1.5)$ for $E=k^{2}$ and $\psi_{-\gamma}^{0}(x,-k,-l)$ satisfies (1.5) with $v$ replaced by $v^{0}$, for $E=k^{2}=l^{2}$. Thus, to prove Proposition 1 , it remains to prove (3.11).

To prove (3.11) we use, in particular, that

$$
\psi_{\gamma}(x, k, l)=\psi_{\gamma}^{0}(x, k, l)+\int_{\mathbb{R}^{d}} R_{\gamma}^{0}(x, y, k)\left(v(y)-v^{0}(y)\right) \psi_{\gamma}(y, k, l) d y
$$

for $x \in \mathbb{R}^{d}, \gamma \in \mathbb{S}^{d-1}, k \in \mathbb{R}^{d} \backslash\left(0 \cup \mathcal{E}_{\gamma}^{0} \cup \mathcal{E}_{\gamma}\right), l \in \mathbb{R}^{d}, k^{2}=l^{2}$. To obtain (3.12) we write $(2.14 \mathrm{a})$ as

$$
\begin{aligned}
& \psi_{\gamma}(x, k, l)-\int_{\mathbb{R}^{d}} G_{\gamma}(x-y, k) v^{0}(y) \psi_{\gamma}(y, k, l) d y-e^{i l x}= \\
& \int_{\mathbb{R}^{d}} G_{\gamma}(x-y, k)\left(v(y)-v^{0}(y)\right) \psi_{\gamma}(y, k, l) d y,
\end{aligned}
$$

where $x \in \mathbb{R}^{d}, \gamma \in \mathbb{S}^{d-1}, k \in \mathbb{R}^{d} \backslash\left(0 \cup \mathcal{E}_{\gamma}\right), l \in \mathbb{R}^{d}, k^{2}=l^{2}$. Replacing $e^{i l x}$ in (3.13) by its expression from (2.14a) with $v$ and $\psi_{\gamma}$ replaced by $v^{0}$ and $\psi_{\gamma}^{0}$ we have

$$
\begin{aligned}
& \psi_{\gamma}(x, k, l)-\psi_{\gamma}^{0}(x, k, l)-\int_{\mathbb{R}^{d}} G_{\gamma}(x-y, k) v^{0}(y)\left(\psi_{\gamma}(y, k, l)-\psi_{\gamma}^{0}(y, k, l)\right) d y= \\
& \int_{\mathbb{R}^{d}} G_{\gamma}(x-y, k)\left(v(y)-v^{0}(y)\right) \psi_{\gamma}(y, k, l) d y,
\end{aligned}
$$


where $x \in \mathbb{R}^{d}, \gamma \in \mathbb{S}^{d-1}, k \in \mathbb{R}^{d} \backslash\left(0 \cup \mathcal{E}_{\gamma}^{0} \cup \mathcal{E}_{\gamma}\right), l \in \mathbb{R}^{d}, k^{2}=l^{2}$.

Comparing (3.14) (as an equation for $\psi_{\gamma}-\psi_{\gamma}^{0}$ ) with the following equation (arising from $(2.2),(2.5),(2.14 \mathrm{~b}))$ for $R_{\gamma}^{0}(x, y, k)$ :

$$
R_{\gamma}^{0}(x, y, k)-\int_{\mathbb{R}^{d}} G_{\gamma}(x-z, k) v^{0}(z) R_{\gamma}^{0}(z, y, k) d z=G_{\gamma}(x-y, k),
$$

$x, y \in \mathbb{R}^{d}, \gamma \in \mathbb{S}^{d-1}, k \in \mathbb{R}^{d} \backslash\left(0 \cup \mathcal{E}_{\gamma}^{0} \cup \mathcal{E}_{\gamma}\right)$, we obtain (3.12).

Note that for $v \equiv 0$ equation (3.12) with $k, \gamma$ and $l$ replaced by $-k,-\gamma$ and $-l$ takes the form

$$
e^{-i l x}=\psi_{-\gamma}^{0}(x,-k,-l)-\int_{\mathbb{R}^{d}} R_{-\gamma}^{0}(x, y,-k) v^{0}(y) e^{-i l y} d y,
$$

$x \in \mathbb{R}^{d}, \gamma \in \mathbb{S}^{d-1}, k \in \mathbb{R}^{d} \backslash\left(0 \cup \mathcal{E}_{\gamma}^{0}\right)$. To prove (3.11) we will use also the following symmetry (following from (3.10))

$$
R_{\gamma}(x, y, k)=R_{-\gamma}(y, x,-k), \quad x, y \in \mathbb{R}^{d}, \quad \gamma \in \mathbb{S}^{d-1}, \quad k \in \mathbb{R}^{d} \backslash 0 .
$$

The following sequences of equalities proves (3.11):

$$
\begin{aligned}
& (2 \pi)^{d} h_{\gamma}(k, l)=\int_{\mathbb{R}^{d}} e^{-i l x} v(x) \psi_{\gamma}(x, k) d x=\int_{\mathbb{R}^{d}} e^{-i l x} v^{0}(x) \psi_{\gamma}(x, k) d x+ \\
& \int_{\mathbb{R}^{d}} e^{-i l x}\left(v(x)-v^{0}(x)\right) \psi_{\gamma}(x, k) d x \stackrel{(3.16)}{=} \int_{\mathbb{R}^{d}} e^{-i l x} v^{0}(x) \psi_{\gamma}(x, k) d x+ \\
& \int_{\mathbb{R}^{d}} \psi_{-\gamma}^{0}(x,-k,-l)\left(v(x)-v^{0}(x)\right) \psi_{\gamma}(x, k) d x- \\
& \int_{\mathbb{R}^{d}} \int_{\mathbb{R}^{d}} R_{-\gamma}^{0}(x, y,-k) v^{0}(y) e^{-i l y}\left(v(x)-v^{0}(x)\right) \psi_{\gamma}(x, k) d y d x \stackrel{(3.17)}{=} \\
& \int_{\mathbb{R}^{d}} e^{-i l x} v^{0}(x) \psi_{\gamma}(x, k) d x+\int_{\mathbb{R}^{d}} \psi_{-\gamma}^{0}(x,-k,-l)\left(v(x)-v^{0}(x)\right) \psi_{\gamma}(x, k) d x- \\
& \int_{\mathbb{R}^{d}} \int_{\mathbb{R}^{d}} R_{\gamma}^{0}(y, x, k) v^{0}(y) e^{-i l y}\left(v(x)-v^{0}(x)\right) \psi_{\gamma}(x, k) d x d y \stackrel{(3.4)}{=} \\
& \int_{\mathbb{R}^{d}} e^{-i l x} v^{0}(x) \psi_{\gamma}(x, k) d x+\int_{\mathbb{R}^{d}} \psi_{-\gamma}^{0}(x,-k,-l)\left(v(x)-v^{0}(x)\right) \psi_{\gamma}(x, k) d x+ \\
& \int_{\mathbb{R}^{d}} e^{-i l y} v^{0}(y)\left(\psi_{\gamma}^{0}(y, k)-\psi_{\gamma}(y, k)\right) d y= \\
& (2 \pi)^{d} h_{\gamma}^{0}(k, l)+\int_{\mathbb{R}^{d}} \psi_{-\gamma}^{0}(x,-k,-l)\left(v(x)-v^{0}(x)\right) \psi_{\gamma}(x, k) d x
\end{aligned}
$$

for $\gamma \in \mathbb{S}^{d-1}, k \in \mathbb{R}^{d} \backslash\left(0 \cup \mathcal{E}_{\gamma}^{0} \cup \mathcal{E}_{\gamma}\right), l \in \mathbb{R}^{d}, k^{2}=l^{2}$.

Proposition 1 is proved.

Proof of Proposition 2. Let us prove Proposition 2 for the case of equation (2.9a). Consider the operator $A(k)$ of $(2.9 \mathrm{a})$ :

$$
A(k)=R^{0}(k)\left(\Phi(E)-\Phi^{0}(E)\right),
$$


where $k \in \mathbb{C}^{d} \backslash\left(\mathbb{R}^{d} \cup \mathcal{E}^{0}\right), k^{2}=E$, and where the operator $R^{0}(k)$ is defined by

$$
R^{0}(k) \varphi(x)=\int_{\partial D} R^{0}(x, y, k) \varphi(y) d y, \quad x \in \partial D
$$

where $\varphi$ is a test function.

Under the assumptions of Theorem 1, the operator $\Phi(E)-\Phi^{0}(E)$ is compact in $L^{\infty}(\partial D)$. This follows from the following properties of the Schwartz kernel of $\Phi(E)-\Phi^{0}(E)$ :

$$
\begin{gathered}
\left(\Phi-\Phi^{0}\right)(x, y, E) \text { is continuous for } x, y \in \partial D, \quad x \neq y, \\
\left|\left(\Phi-\Phi^{0}\right)(x, y, E)\right| \leq C_{1}|x-y|^{2-d}, \quad x, y \in \partial D, \quad \text { for } d \geq 3, \\
\left|\left(\Phi-\Phi^{0}\right)(x, y, E)\right| \leq C_{1}|\ln | x-y||, \quad x, y \in \partial D, \quad \text { for } d=2,
\end{gathered}
$$

where $C_{1}$ is some constant (dependent on $D, v, v^{0}, E$ and $d$ ). Note that for $v^{0} \equiv 0$ the result that $\Phi(E)-\Phi^{0}(E)$ is compact in $L^{\infty}(\partial D)$ (under the assumptions of Theorem 1) was given in [No1].

If (1.2a) is fulfilled, $v^{0}$ satisfies (1.15) and $k \in \mathbb{C}^{d} \backslash\left(\mathbb{R}^{d} \cup \mathcal{E}^{0}\right)$, then $R^{0}(k)$ is a compact operator in $L^{\infty}(\partial D)$. This follows already from the following properties of $R^{0}(x, y, k)$ :

$$
\begin{gathered}
R^{0}(x, y, k) \text { is continuous for } x, y \in \bar{D}, \quad x \neq y, \\
\left|R^{0}(x, y, k)\right| \leq C_{2}|x-y|^{2-d}, \quad x, y \in \bar{D}, \text { for } d \geq 3, \\
\left|R^{0}(x, y, k)\right| \leq C_{2}|\ln | x-y||, \quad x, y \in \bar{D}, \quad \text { for } d=2,
\end{gathered}
$$

where $C_{2}$ is some constant (dependent on $D, v^{0}, k$ and $d$ ).

Actually, under the aforementioned assumptions on $D, v^{0}$ and $k, R^{0}(k)$ is a bounded operator from $L^{\infty}(\partial D)$ to $C^{\alpha}(\partial D)$ for any $\alpha \in\left[0,1\left[\right.\right.$ (where $C^{\alpha}$ denotes the Hölder space). This result in the general case follows from this result for $v^{0} \equiv 0$ (when $R^{0}(x, y, k)=$ $G(x-y, k))$, the relation $(2.2)$, the estimate (3.23) and the property that

$$
\begin{aligned}
& \int_{D} G(x-z, k) u(z) d z \in C^{\alpha}\left(\mathbb{R}^{d}\right), \text { at least, for any } \alpha \in[0,1] \\
& \text { (as a function of } x \text { ) for } u \in L^{\infty}(D) \text { (and } k \in \mathbb{C}^{d} \backslash \mathbb{R}^{d} \text { ). }
\end{aligned}
$$

The formula (3.18) and the aforementioned properties of the operators $\Phi(E)-\Phi^{0}(E)$ and $R^{0}(k)$ imply that, under the assumptions of Theorem 1 , for fixed $k \in \mathbb{C}^{d} \backslash\left(\mathbb{R}^{d} \cup \mathcal{E}^{0}\right)$, $k^{2}=E, A(k)$ is a compact operator in $L^{\infty}(\partial D)$ and, thus, (2.9a) is a Fredholm linear integral equation of the second kind for $\psi$ in $L^{\infty}(\partial D)$.

Under the assumptions of Theorem 1 , for $k \in \mathbb{C}^{d} \backslash\left(\mathbb{R}^{d} \cup \mathcal{E}^{0}\right), k^{2}=E$, the aforementioned properties of $\Phi(E)-\Phi^{0}(E)$ and $R^{0}(k)$ and the property that $\psi^{0} \in C^{\alpha}(\partial D)$, $\alpha \in[0,1[$, imply also that

$$
\text { if } \psi \in L^{\infty}(\partial D) \text { satisfies }(2.9 a) \text {, then } \psi \in C^{\alpha}(\partial D) \text { for any } \alpha \in[0,1[\text {. }
$$


Note also that if (1.2a) is fulfilled, $v^{0}$ satisfies (1.15) and $k \in \mathbb{C}^{d} \backslash\left(\mathbb{R}^{d} \cup \mathcal{E}^{0}\right)$, then $R^{0}(k)$ is a bounded operator from $L^{2}(\partial D)$ to $H^{1}(\partial D)$. One can prove this result in the general case proceeding from this result for $v^{0} \equiv 0$ (when $R^{0}(x, y, k)=G(x-y, k)$ ), equation (2.2) with its iterations for $R(x, y, k), R(x, y, k)-G(x-y, k)$ and so on, estimate (3.23) and the property that

$$
\int_{D} G(x-z, k) u(z) d z \in H^{2}(D) \quad \text { (as a function of } x \text { ) for } u \in L^{2}(D) \quad\left(\text { and } k \in \mathbb{C}^{d} \backslash \mathbb{R}^{d}\right. \text { ). }
$$

(For $v^{0} \equiv 0$ this result was given, for example, in [Na1].) Therefore, under the assumptions of Theorem 1, for $k \in \mathbb{C}^{d} \backslash\left(\mathbb{R}^{d} \cup \mathcal{E}^{0}\right), k^{2}=E$, one can see that

$$
\text { if } \psi \in L^{\infty}(\partial D) \text { satisfies }(2.9 a) \text {, then also } \psi \in H^{1}(\partial D) \text {. }
$$

To prove Proposition 2 for the case of equation (2.9a) it remains to show that, under the assumptions of Theorem 1, equation (2.9a) at fixed $k \in \mathbb{C}^{d} \backslash\left(\mathbb{R}^{d} \cup \mathcal{E}^{0}\right), k^{2}=E$, is uniquely solvable for $\psi \in L^{\infty}(\partial D)$ iff $k \notin \mathcal{E}$.

For $k \in \mathbb{C}^{d} \backslash\left(\mathbb{R}^{d} \cup \mathcal{E}^{0}\right)$, under the assumption (1.6) for $v$ and $v^{0}$, due to (2.1a) and (3.7), $k \notin \mathcal{E}$ iff equation (3.2) is uniquely solvable for $\psi=e^{i k x} \mu(x, k)$ with $\mu \in L^{\infty}\left(\mathbb{R}^{d}\right)$. In turn, for $k \in \mathbb{C}^{d} \backslash\left(\mathbb{R}^{d} \cup \mathcal{E}^{0}\right)$, under the assumption (1.15) for $v$ and $v^{0}$, equation (3.2) is uniquely solvable for $\psi=e^{i k x} \mu(x, k)$ with $\mu \in L^{\infty}\left(\mathbb{R}^{d}\right)$ iff (3.2) is uniquely solvable for $\psi \in C\left(\mathbb{R}^{d}\right)$. Thus, it remains to show that, under the assumptions of Theorem 1, equation (2.9a) at fixed $k \in \mathbb{C}^{d} \backslash\left(\mathbb{R}^{d} \cup \mathcal{E}^{0}\right), k^{2}=E$, is uniquely solvable for $\psi \in L^{\infty}(\partial D)$ iff $(3.2)$ is uniquely solvable for $\psi \in C\left(\mathbb{R}^{d}\right)$. This proof consists of the following two parts.

Part 1. Suppose that (under the assumptions of Theorem 1) at fixed $k \in \mathbb{C}^{d} \backslash\left(\mathbb{R}^{d} \cup \mathcal{E}^{0}\right)$, $k^{2}=E$, equation (3.2) has several solutions in $C\left(\mathbb{R}^{d}\right)$. Then, repeating the proof of $(2.9)$ separately for each solution, we find that the restriction to $\partial D$ of each of these solutions satisfies (2.9a). In addtion, different solutions $\psi$ have different restrictions to $\partial D$. This follows from (1.3). Thus at fixed $k \in \mathbb{C}^{d} \backslash\left(\mathbb{R}^{d} \cup \mathcal{E}^{0}\right), k^{2}=E$, equation (2.9a) has, at least, as many solutions as equation (3.2).

Part 2. To prove the converse, we use the following identities:

$$
\begin{gathered}
\int_{D} R^{0}(x, y, k)\left(v(y)-v^{0}(y)\right) \psi(y) d y \stackrel{(1.1)}{=} \int_{D} R^{0}(x, y, k)(\Delta+E) \psi(y) d y- \\
\int_{D} R^{0}(x, y, k) v^{0}(y) \psi(y) d y \stackrel{(3.8)}{=} \int_{D} \psi(y)\left(\Delta_{y}+E-v^{0}(y)\right) R^{0}(x, y, k) d y+ \\
\int_{\partial D}\left(R^{0}(x, y, k) \frac{\partial}{\partial \nu_{y}} \psi(y)-\psi(y) \frac{\partial}{\partial \nu_{y}} R^{0}(x, y, k)\right) d y \stackrel{(2.7),(3.10)}{=} \\
\int_{D} \psi(y) \delta(x-y) d y+\int_{\partial D}\left(R^{0}(x, y, k) \frac{\partial}{\partial \nu_{y}} \psi(y)-\psi(y) \frac{\partial}{\partial \nu_{y}} R^{0}(x, y, k)\right) d y \\
\quad \text { for } x \in \mathbb{R}^{d} \backslash \partial D \\
\int_{D} R^{0}(x, y, k)\left(v(y)-v^{0}(y)\right) \psi(y) d y \stackrel{(3.9),(2.9 b)}{=} \int_{\partial D} A(x, y, k) \psi(y) d y \text { for } x \in \mathbb{R}^{d} \backslash \bar{D},
\end{gathered}
$$


where $k \in \mathbb{C}^{d} \backslash\left(\mathbb{R}^{d} \cup \mathcal{E}^{0}\right), k^{2}=E, \psi$ satisfies (1.1) and is sufficiently regular in $D$, for example, $\psi \in H^{1}(D)$.

Let $k \in \mathbb{C}^{d} \backslash\left(\mathbb{R}^{d} \cup \mathcal{E}^{0}\right), k^{2}=E$, be fixed. Suppose that $\psi \in L^{\infty}(\partial D)$ solves (2.9a). Due to (3.24), (3.25), we have also that $\psi \in C^{\alpha}(\partial D), \alpha \in\left[0,1\left[\right.\right.$, and $\psi \in H^{1}(\partial D)$. Consider this $\psi$ as Dirichlet data for equation (1.1) and consider the solution $\psi$ (of (1.1)) corresponding to these data. We have that $\psi \in C^{\alpha}(\bar{D}), \alpha \in\left[0,1\left[\right.\right.$, and $\psi \in H^{3 / 2}(D)$.

Let $\psi$ be also defined on $\mathbb{R}^{d} \backslash \bar{D}$ by (2.9) with $x \in \mathbb{R}^{d} \backslash \bar{D}$ (in terms of $\left.\psi\right|_{\partial D}$ ). Let us prove that $\psi$ defined in such a way on $\mathbb{R}^{d}=\partial D \cup D \cup\left(\mathbb{R}^{d} \backslash \bar{D}\right)$ satisfies (3.2) and belongs to $C\left(\mathbb{R}^{d}\right)$. As a particular case of the aforementioned property $\psi \in C^{\alpha}(\bar{D}), \alpha \in[0,1[$, we have that $\psi \in C(\bar{D})$. Proceeding from the definition of $\psi$ on $\mathbb{R}^{d} \backslash \bar{D}$, one can easily show that, at least, $\psi \in C\left(\mathbb{R}^{d} \backslash D\right)$. The properties $\psi \in C(\bar{D})$ and $\psi \in C\left(\mathbb{R}^{d} \backslash D\right)$ imply that $\psi \in C\left(\mathbb{R}^{d}\right)$. The proof that $\psi$ satisfies (3.2) consists in the following. First, from (2.9) with $x \in \mathbb{R}^{d} \backslash \bar{D}$ and (3.27) (and the continuity of $\psi$ ) we obtain that $\psi$ satisfies (3.2) for $x \in \mathbb{R}^{d} \backslash D$. In addition, taking into account (3.26) we have also that

$$
\psi(x)=\psi^{0}(x, k)+\int_{\partial D}\left(R^{0}(x, y, k) \frac{\partial}{\partial \nu_{y}} \psi(y)-\psi(y) \frac{\partial}{\partial \nu_{y}} R^{0}(x, y, k)\right) d y
$$

for $x \in \mathbb{R}^{d} \backslash \bar{D}$, where $\frac{\partial}{\partial \nu} \psi$ is taken for $\psi$ defined on $\bar{D}$. Further, (as well as for $v^{0} \equiv 0$, see $[\mathrm{No} 1],[\mathrm{Na} 2])$ proceeding from (3.28) we obtain that

$$
\psi(x)=\psi^{0}(x, k)+\psi(x)+\int_{\partial D}\left(R^{0}(x, y, k) \frac{\partial}{\partial \nu_{y}} \psi(y)-\psi(y) \frac{\partial}{\partial \nu_{y}} R^{0}(x, y, k)\right) d y
$$

for $x \in D$, where $\frac{\partial}{\partial \nu} \psi$ is taken for $\psi$ defined in $\bar{D}$. Note that proceeding from (3.28) and using that $\psi \in H^{1}(\partial D), \frac{\partial}{\partial \nu} \psi \in L^{2}(\partial D)$ and the jump property of the double lager potential $\frac{\partial}{\partial \nu_{y}} R^{0}(x, y, k)$, we obtain, first, (3.29) in the limit for $x=\xi-0 \nu_{\xi}, \xi \in \partial D$ (where $\nu_{\xi}$ is the outward normal to $\partial D$ at $\xi$ ). Further, using that $\psi^{0}(x, k)$ and $R^{0}(x, y, k)$ with $\frac{\partial}{\partial \nu_{y}} R^{0}(x, y, k), y \in \partial D$, satisfy (1.1) with $v^{0}$ in place of $v$, for $E=k^{2}$, we obtain (3.29) for $x \in D$. Finally, from (3.29) and (3.26) we obtain that $\psi$ satisfies (3.2) also for $x \in D$. Thus, any solution $\psi$ of $(2.9 \mathrm{a})$ can be continued to a continuous solution of (3.2). This completes the (part 2 of) proof that, under the assumptions of Theorem 1, at fixed $k \in \mathbb{C}^{d} \backslash\left(\mathbb{R}^{d} \cup \mathcal{E}^{0}\right), k^{2}=E$, equation (2.9a) is uniquely solvable for $\psi \in L^{\infty}(\partial D)$ iff $(3.2)$ is uniquely solvable for $\psi \in C\left(\mathbb{R}^{d}\right)$.

The proof of Proposition 2 for the case of equation (2.9a) is completed. The proof of Proposition 2 for the cases of equations (2.11a) and (2.13a) is similar.

\section{References}

[ A] Alessandrini G 1988 Stable determination of conductivity by boundary measurements Appl. Anal. 27 153-172

[ BC] Beals R and Coifman R R 1985 Multidimensional inverse scattering and nonlinear partial differential equations Proc. Symp. Pure Math. 43 45-70 
[BBMRS] Bogatyrev A V, Burov V A, Morozov S A, Rumyantseva O D and Sukhov E G 2000 Numerical realization of algorithm for exact solution of two-dimensional monochromatic inverse problem of acoustical scattering Acoustical Imaging 25 (Kluwer Academic/Plenum Publishers,New York) 65-70

[ BMR] Burov V A, Morozov S A and Rumyantseva O D 2002 Reconstruction of fine-scale structure of acoustical scatterer on large-scale contrast background Acoustical Imaging 26 (Kluwer Academic/Plenum Publishers,New York) 231-238

[ Ch] Chen Yu 1997 Inverse scattering via Heisenberg's uncertainty principle Inverse Problems 13 253-282

[ E] Eskin G 2001 The inverse scattering problem in two dimensions at a fixed energy Comm. Partial Differential Equations 26 1055-1090

[ F1] Faddeev L D 1965 Increasing solutions of the Shrödinger equation Dokl. Akad. Nauk SSSR 165 514-517 (in Russian); English transl.: 1966 Sov. Phys. Dokl. 10 1033-1035

[ F2] Faddeev L D 1966 Factorization of the $S$ matrix for the multidimensional Shrödinger operator Dokl. Akad. Nauk SSSR 167 69-72 (in Russian); English transl.: 1966 Sov. Phys. Dokl. 11 209-211

[ F3] Faddeev L D 1974 Inverse problem of quantum scattering theory II Itogi Nauki Tekhn. Sovrem. Prob. Mat. 3 93-180 (in Russian); English transl.: 1976 Sov. Math. 5 334396

[ GN] Grinevich P G and Novikov R G 1995 Transparent potentials at fixed energy in dimension two. Fixed-energy dispersion relations for the fast decaying potentials Comm. Math. Phys. 174 409-446

[ HN] Henkin G M and Novikov R G 1987 The $\bar{\partial}$ - equation in the multidimensional inverse scattering problem Uspekhi Mat. Nauk 42 93-152 (in Russian); English transl.: 1987 Russ. Math. Surv. 42 109-180

[ IS] Isakov V and Sun Z 1995 The inverse scattering at fixed energies in two dimensions Indiana Univ. Math. J. 44 883-896

[ LN] Lavine R B and Nachman A I 1987 On the inverse scattering transform of the $n$ dimensional Schrödinger operator In Topics in Soliton Theory and Exactly Solvable Nonlinear Equations (M.Ablovitz, B.Fuchssteiner and M.Kruskal, Eds.), pp. 33-44. World Scientific, Singapore

[ M] Mandache N 2001 Exponential instability in an inverse problem for the Schrödinger equation Inverse Problems 17 1435-44

[ Na1] Nachman A I 1988 Reconstructions from boundary measurements Ann. Math. 128 $531-576$

[ Na2] Nachman A I 1995 Global uniqueness for a two-dimensional inverse boundary value problem Ann. Math. 142 71-96

[ NSU] Nachman A I, Sylvester J and Uhlmann G 1988 An n- dimensional Borg-Levinson theorem Comm. Math. Phys. 115 593-605

[ Ne] Newton R G 1989 Inverse Schrödinger scattering in three dimensions Springer-Verlag, Berlin

[ No1] Novikov R G 1988 Multidimensional inverse spectral problem for the equation $-\Delta \psi+$ $(v(x)-E u(x)) \psi=0$. Funkt. Anal. Ego Pril 22 11-22 (in Russian); English transl.: 1988 Funct. Anal. Appl. 22 263-272 
[ No2] Novikov R G 1992 The inverse scattering problem on a fixed energy level for the two-dimensional Schrödinger operator J. Funct. Anal. 103 409-463

[ No3] Novikov R G 1994 The inverse scattering problem at fixed energy for the threedimensional Schrödinger equation with an exponentially decreasing potential Comm. Math. Phys. 161 569-595

[ No4] Novikov R G $1996 \bar{\partial}$-method with nonzero background potential. Application to inverse scattering for the two-dimensional acoustic equation Comm. Partial Differential Equations 21 597-618

[ No5] Novikov R G 1999 Approximate inverse quantum scattering at fixed energy in dimension 2 Proc. Steklov Inst. of Math. 225 285-302

[ No6] Novikov R G 2002 Scattering for the Schrödinger equation in multidimension. Nonlinear $\bar{\partial}$-equation, characterization of scattering data and related results. Chapter 6.2.4 in SCATTERING edited by E.R.Pike and P.Sabatier, Academic Press

[St] Stefanov P 1990 A uniqueness result for the inverse back-scattering problem Inverse Problems 6 1055-1064

[ SU] Sylvester J and Uhlmann G. 1987 A global uniqueness theorem for an inverse boundary value problem Ann. of Math. 125 153-169

[ We] Weder R 1991 Generalized limiting absorption method and multidimensional inverse scattering theory Math. Methods Appl. Sci. 14 509-524 